\title{
Gold Mirrors for X-Ray Astronomy
}

\author{
Michael Cruise \\ Mullard Space Science Laboratory, University College, London
}

The rapidly expanding field of X-ray astronomy promotes the study of celestial bodies that are far hotter than our sun. At temperatures of one to a hundred million degrees Kelvin, most of the radiation is in the form of X-rays rather than visible light. These conditions seem to be produced in the regions surrounding stars that have undergone an explosion and have subsequently collapsed to form the compact objects known as neutron stars or black holes. Since $\mathrm{X}$-rays in the range $100 \mathrm{eV}$ to $50 \mathrm{keV}$ are readily absorbed in the earth's atmosphere, the telescopes that study X-ray stars are always mounted on rockets or satellites orbiting the earth.

In order to reflect and focus the $\mathrm{X}$-rays on to the detectors a paraboloid of revolution is used; to achieve a high reflection efficiency, however, two conditions must be met. First, the surface must be extremely smooth with r.m.s. height variations below about $20 \AA$, and secondly the $\mathrm{X}$-rays must strike the reflecting surface at a very small grazing angle.

The reflection process is analogous to the total internal reflection experienced at visible wavelengths with the exception that, since the real part of the refractive index is less than unity at X-ray frequencies, the total reflection is experienced when radiation travels from vacuum into a medium of higher density. The reflection efficiency is strongly dependent on the wavelength of the X-rays, the angle at which they strike the surface, and the surface material itself.

One of the four X-ray mirrors that will fly on UK-6, Britain's next satellite devoted to astronomical observations, is shown here. Due for launch early next year, the satellite will carry an $\mathrm{X}$-ray observatory complete with its own data handling computer, all of which can be controlled from the ground. The instrument containing the telescopes is being designed and built at the Mullard Space Science Laboratory of University College, London.

The four mirrors, and a set of four spares, have been manufactured by Ion Tech Limited of Teddington, Middlesex. Nickel was electrodeposited on to the aluminium alloy mirror blank and this surface was then polished to the very high standard required by a process developed at the National Physical Laboratory. A gold coating was then superimposed on the nickel by Ion Tech under very carefully controlled conditions.

Gold was chosen as the surface material because it has good reflection properties at these wavelengths and does not show any sharp absorption edges which complicate the reconstruction of the stars' X-ray spectrum during analysis of the data. The nickel layer is easier to polish than the gold and forms a very stable substrate, but its $\mathrm{X}$-ray properties are not as attractive in this application.

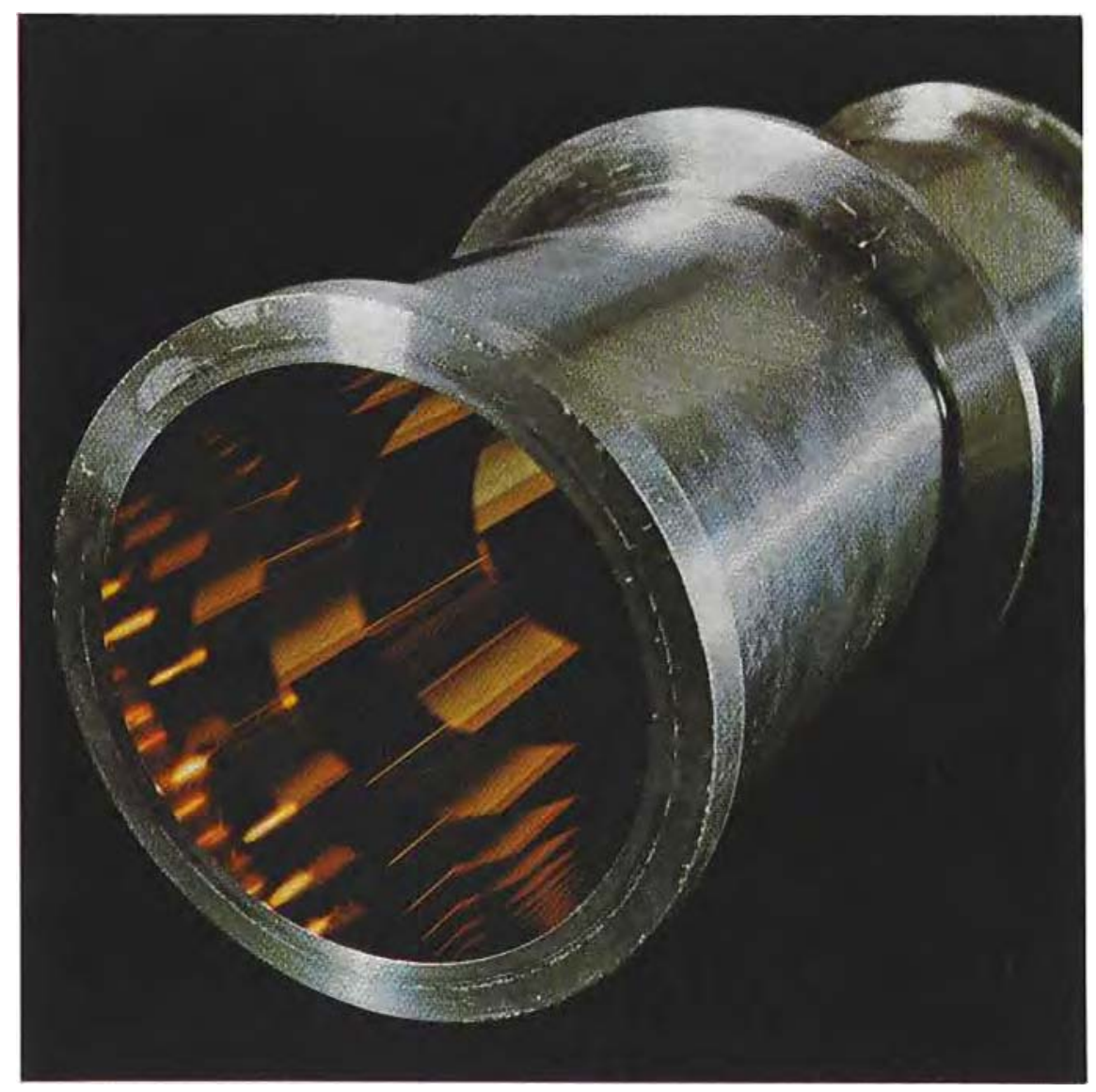

